\title{
Avaliação postural de sURfistas PROfissionaIS Utilizando o método PortLANd State University (PSU)
}

\author{
Rosemeri Peirão ${ }^{1,2,3}$ rosemerip@yahoo.com.br \\ Adriana Seára Tirloni ${ }^{3,4}$ adri@tirloni.com.br \\ Diogo Cunha dos Reis ${ }^{3,4,5}$ diogo.biomecanica@gmail.com
}

doi:10.3900/fpj.7.6.370.p

Peirão R, Tirloni AS, Reis DC. Avaliação postural de surfistas profissionais utilizando o método Portland State University (PSU). Fit Perf J. 2008 nov-dez; $7(6): 370-4$

\section{RESUMO}

Introdução: Apesar da popularidade do surfe, ainda são escassos estudos referentes às conseqüências advindas da prática desse esporte. $\bigcirc$ objetivo deste estudo descritivo foi identificar quais as incidências de desvios posturais em surfistas profissionais e se elas têm relação com o tempo de prática do surfe e com o tempo como profissionais.

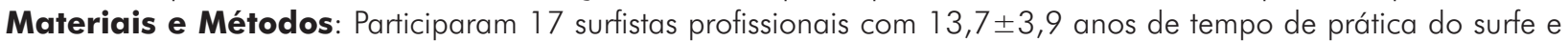
3,9 4, 1 anos como surfistas profissionais. Para a avaliação postural foi utilizado o método Portland State University (PSU) e para o tratamento estatístico utilizou-se a correlação de Spearman e Pearson ( $\mathrm{p} \leq 0,05)$. Resultados: Apesar de 70,6\% dos atletas terem apresentado desvio lateral de ombros, 94,1\% ombros projetados anteriormente, 70,6\% aumento da curvatura lombar e 76,5\% joelhos hiperestendidos, esses desvios não são considerados relevantes segundo o método PSU, já que a média do Índice de Correção Postural (ICP) foi de 86,6 5,0\% (níveis considerados como boa postura). O tempo de prática do surfe e o tempo como profissional não apresentaram correlação significativa com os desvios encontrados e com o ICP. Discussão: Embora a análise postural dos surfistas utilizando o PSU não tenha apresentado desvios posturais acentuados, observaram-se assimetrias que provavelmente podem ser decorrentes das características da própria modalidade.

\section{PALAVRAS-CHAVE}

Coluna Vertebral, Postura, Dor Lombar.

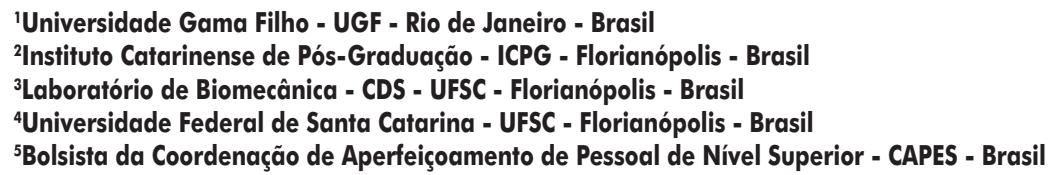

Copyright(C 2008 por Colégio Brasileiro de Atividade Física, Saúde e Esporte

Fit Perf J | Rio de Janeiro | 7 | 6 | 370-374 | nov/dez 2008 
Postural evaluation of professional surfers using the Portland State University method (PSU)

\section{ABSTRACT}

Introduction: Despite the popularity of surfing, studies on the consequences of the practice of sport are still scarce. This study descriptive aimed to identify the incidences of professional surfers' postural deviations and whether there is a relationship of these deviations with years of surfing practice and time as professionals or not. Materials and Methods: The sample was composed by 17 professional surfers with an average of $13.7 \pm 3.9$ years of practice and $3.9 \pm 4.1$ years as professional surfers. The postural assessment used the Portland State University (PSU) method and as statistical treatment, correlation of Spearman and Pearson were tested ( $\mathrm{p} \leq 0.05)$. Results: Even though $70.6 \%$ of the athletes had lateral deviation of shoulders, 94.1\% shoulders projected forward, $70.6 \%$ increased lumbar curvature and $76.5 \%$ hyper extended knees, these deviations are not considered relevant by the PSU method, as the average Posture Correction Index (PCI) was $86.6 \pm 5.0 \%$ (classified as a good posture). The years of surfing practice as well as time as a professional, showed no significant correlation with the deviations found and the PCl. Discussion: Although the analysis posture of surfers using the PSU has not presented great postural deviations, it was found asymmetries that may be considered characteristics of the modality.

\section{KEYWORDS}

Spine, Posture, Low Back Pain.

Postural evaluación de surfistas profesionales utilizando el método de Portland State University (PSU)

\section{RESUMEN}

Introducción: A pesar de la popularidad de surf, aún son escasos los estudios de las consecuencias derivadas de la práctica del deporte. El objetivo del estudio descriptivo fue determinar los efectos de las desviaciones posturales en los surfistas profesionales y si se relacionan con el momento de la práctica de surf y con el tiempo como profesionales. Materiales y Métodos: Participaran 17 surfistas profesionales con 13,7 $\pm 3,9$ años de tiempo para la práctica de surf y $3,9 \pm 4,1$ años como profesional. Para la evaluación de la postura se utilizó lo método Portland State University (PSU) y el tratamiento estadístico se utilizaron las correlación de Spearman y Pearson ( $\mathrm{p} \leq 0,05)$. Resultados: Aunque el 70,6\% de los atletas había desviación lateral de hombros, 94,1\% hombros proyectado anteriormente, $70,6 \%$ un aumento del curvatura lumbar y $76,5 \%$ rodillas hiperestendidos, estas desviaciones no se han considerado pertinentes de lo método PSU, ya que el promedio del Índice de Corrección de Postura (ICP) fue 86,6 $\pm 5,0 \%$ (niveles considerados una buena postura). La práctica del surf cada cierto tiempo como un profesional y no mostró correlación significativa con las desviaciones encontradas y el ICP. Discusión: Aunque el análisis de la postura de los surfistas utilizando el PSU no ha presentado desviaciones posturales acentuados, hubo probablemente asimetrías que pueden ser el resultado de las características de la modalidad.

\section{PALABRAS-CLAVE}

Columna Vertebral, Postura, Dolor de la Región Lumbar.

\section{INTRODUÇÃO}

Diversos pesquisadores afirmam que o surfe é um esporte completo por ser considerado uma atividade aeróbica e anaeróbica de moderada a alta intensidade ${ }^{\mathbf{1 , 2 , 3}}$ Para alcançar o estágio que é conhecido hoje, o surfe passou por um processo histórico de transformação onde poucos fatos, inclusive sua origem, foram registrados. Os historiadores indicam dois pontos em especial que contribuíram com o seu estado atual. O primeiro marcado pela sua prática entre os povos peruanos, polinésios e havaianos e o segundo pelo seu redescobrimento no século XX e marcado pela figura de Duke Paoa Kahanamoku (1890-1968), perdurando até os dias atuais ${ }^{\mathbf{1}, 4,5}$.

Apesar de toda a popularidade do surfe ao nível mundial, ainda são escassos estudos referentes às conseqüências advindas da prática desse esporte e os fatores que prejudicam e os que otimizam a performance dos praticantes e, principalmente, dos atletas' ${ }^{1}$. Alguns estudos vêm abordando os cuidados com as lesões agudas de- correntes da prática do surfe ${ }^{6,7}$. Taylor et al. ${ }^{8}$ analisaram a incidência de lesões crônicas, durante um período de doze meses, em 646 surfistas que tinham em média 10 anos de prática da modalidade. Constataram que 29, 15, 12 e um surfista apresentaram dor ou rigidez nas regiões da cabeça, costas, ombros, joelhos e cotovelos, respectivamente. Nathanson et al. ${ }^{9}$ constataram que, dos 477 casos de lesões crônicas em surfistas, 18\%, 16\%, 9\%, 9\% e 5\% possuíam tensão nos ombros, nas costas, no pescoço, nos joelhos e nos cotovelos, respectivamente. Com o objetivo de verificar a incidência de desvios posturais em surfistas amadores, Peirão et al..$^{10}$ encontraram uma incidência de desvio anterior de ombros, hiperextensão dos joelhos e desvio lateral de ombros e de quadril, constatando que essas assimetrias eram provavelmente decorrentes das características da própria modalidade, como os movimentos realizados durante a prática do esporte (remada e manobras sobre a prancha), assim como pela posição dos pés adotada pelos surfistas sobre a prancha. 
O principal enfoque da avaliação postural está centrado na análise da postura corporal de indivíduos (escolares, atletas, trabalhadores, mulheres grávidas, idosos e portadores de necessidades especiais), diagnosticando os desvios posturais e identificando as regiões mais acometidas"1. $\mathrm{O}$ esporte de alto rendimento determina padrões corporais que freqüentemente extrapolam a linha da saúde, e que possivelmente, a longo prazo, podem resultar em alterações posturais, limitando a prática da modalidade esportiva ${ }^{12}$. Para tal, pesquisadores recomendam exercícios de compensação, pois desenvolvem a força muscular e a flexibilidade para manter uma postura corporal adequada. Tão importante quanto o desenvolvimento das qualidades específicas para o alto desempenho, deve ser a preocupação com a postura e o equilíbrio muscular, pois estes influenciam no rendimento do atleta e podem minimizar a incidência de lesões ${ }^{13}$.

Entre outros fatores, as alterações posturais são resultantes da prática desportiva de alto nível| ${ }^{14,15}$. De acordo com a literatura, as alterações posturais nos atletas ocorrem devido ao treinamento intenso e repetitivo da modalidade esportiva, proporcionando hipertrofia muscular e diminuição da flexibilidade, causando um desequilíbrio entre a musculatura agonista e a antagonista, favorecendo, dessa forma, a instalação de alterações posturais e, por fim, produzindo um resultado estético específico da modalidade ${ }^{16}$.

Dessa forma, este estudo objetivou identificar quais as incidências de desvios posturais em surfistas profissionais, utilizando o método Portland State University (PSU), e se elas têm relação com o tempo de prática do surfe e com o tempo como profissionais na modalidade.

\section{MATERIAIS E MÉTODOS}

Para a realização do presente estudo foram atendidas as exigências legais conforme o que dispõem as Resoluções 196/96 e 251/97 do Conselho Nacional da Saúde, onde o sujeito do estudo, depois de devidamente informado, assinou um Termo de Consentimento Livre e Esclarecido.

Esta pesquisa caracterizou-se como descritiva, do tipo estudo de caso e os participantes foram selecionados de maneira não-probabilística intencional por voluntariado. Avaliou-se a postura corporal estática de 17 surfistas profissionais do sexo masculino, com média de idade de $22,1 \pm 3,7$ anos, estatura de $1,7 \pm 0,1 \mathrm{~m}$ e massa corporal de $67,9 \pm 6,3 \mathrm{~kg}$. Esses atletas possuíam 13,7 $\pm 3,9$ anos de prática do surfe, 3,9 $\pm 4,1$ anos como surfistas profissionais, e treinavam $4,5 \pm 1,4$ h ao dia.

Os dados foram coletados durante dois campeonatos de surfe profissional (WQS - "Mormaii Costão PRO 2007" e "Circuito Cerveja Sol Surf PRO"), nos meses de janeiro e fevereiro de 2007, na praia do Santinho e na praia Mole em Florianópolis - SC. Os instrumentos utilizados para a coleta dos dados, foram: uma máquina fotográfica digital
OLYMPUS ${ }^{\circledR}$ modelo D-395, um tripé e um fio de prumo. Utilizou-se o método de avaliação postural Portland State University (PSU) adaptado por Althoff, Heyden e Robertson (1988). Esse método permite, por meio da observação, quantificar o Índice de Correção Postural (ICP) do avaliado, utilizando-se equações matemáticas (total e por regiões), detectando as assimetrias e os possíveis desvios posturais entre os segmentos corporais. Para tal, este método utiliza como critério três escalas com respectivas pontuações ( 1 - desvio acentuado, 3 - ligeiro desvio e 5 - sem desvio), que são utilizados para pontuar as posturas dos segmentos corporais (treze no total), baseados em figuras-modelos. Os conjuntos dos segmentos corporais formam as seguintes regiões: RCP - região da cabeça e do pescoço, RCDL - região da coluna dorsal e lombar, RAQ - região do abdômen e quadril, RMI - região de membros inferiores e ICP - índice de correção postural. Segundo as recomendações do método, indivíduos adultos com ICP acima de $65 \%$ possuem uma boa postura corporal ${ }^{11}$.

Para a realização da avaliação da postura corporal, primeiramente os indivíduos assinaram o Termo de Consentimento Livre e Esclarecido e, em seguida, cada indivíduo se posicionou no local determinado a uma distância de $3 \mathrm{~m}$ da câmera fotográfica, para registro de uma fotografia em cada posição (lateral e frontal).

As fotografias foram tratadas no programa Corel Draw ${ }^{\circledR}$ 10 for Windows ${ }^{\circledR}$, as pontuações foram tabuladas no programa Exce ${ }^{\circledR}$ for Windows ${ }^{\circledR}$ para a aquisição do Índice de Correção Postural (ICP) e, posteriormente, os testes estatísticos

\begin{tabular}{|c|c|c|c|c|c|}
\hline atleta & RCP & RCDL & RAQ & RMI & ICP \\
\hline 1 & 76,0 & 100,0 & 100,0 & 73,3 & 85,7 \\
\hline 2 & 68,0 & 100,0 & 100,0 & 86,7 & 85,7 \\
\hline 3 & 92,0 & 60,0 & 86,7 & 86,7 & 91,4 \\
\hline 4 & 84,0 & 73,3 & 86,7 & 86,7 & 82,9 \\
\hline 5 & 92,0 & 100,0 & 86,7 & 100,0 & 94,3 \\
\hline 6 & 92,0 & 100,0 & 100,0 & 100,0 & 97,1 \\
\hline 7 & 76,0 & 100,0 & 100,0 & 86,7 & 88,6 \\
\hline 8 & 84,0 & 100,0 & 73,3 & 86,7 & 85,7 \\
\hline 9 & 84,0 & 86,7 & 86,7 & 73,3 & 82,9 \\
\hline 10 & 92,0 & 86,7 & 86,7 & 86,7 & 88,6 \\
\hline 11 & 76,0 & 100,0 & 86,7 & 73,3 & 82,9 \\
\hline 12 & 84,0 & 100,0 & 60,0 & 73,3 & 80,0 \\
\hline 13 & 92,0 & 100,0 & 86,7 & 86,7 & 91,4 \\
\hline 14 & 68,0 & 86,7 & 86,7 & 86,7 & 80,0 \\
\hline 15 & 92,0 & 86,7 & 73,3 & 73,3 & 82,9 \\
\hline 16 & 84,0 & 86,7 & 86,7 & 86,7 & 85,7 \\
\hline 17 & 76,0 & 73,3 & 73,3 & 86,7 & 77,1 \\
\hline média & 83,5 & 91,7 & 86,7 & 84,2 & 86,6 \\
\hline$d p$ & 8,5 & 11,8 & 10,9 & 8,7 & 5,0 \\
\hline
\end{tabular}

$R C P$ - Região cabeça e pescoço; RCDL - Região coluna dorsal e lombar; RAQ - Região abdominal e quadril; RMI - Região membros inferiores; ICP - Índice de correção postural 
foram realizados no programa SPSS ${ }^{\circledR}$. Foi utilizada a estatística descritiva e os dados foram apresentados em forma de média, desvio-padrão e percentual. Para o tratamento estatístico das variáveis foi testada a normalidade dos dados através do teste de Shapiro-Wilk e utilizados os testes de correlação de Spearman e Pearson. Adotou-se o nível de significância de 0,05.

\section{RESULTADOS}

Os resultados do método de avaliação postural PSU para cada indivíduo estão apresentados na Tabela 1 .

A média do ICP dos surfistas profissionais obtida neste estudo $(86,6 \pm 5,0 \%)$ encontra-se dentro dos níveis considerados como boa postura. Apesar de 70,6\% dos atletas terem apresentado desvio lateral de ombros, $94,1 \%$ ombros projetados anteriormente, $70,6 \%$ aumento da curvatura lombar e 76,5\% joelhos hiperestendidos, esses desvios existentes não são considerados relevantes, segundo o método PSU.

Os resultados estatísticos mostraram que o tempo de prática do surfe não apresentou correlação significativa com o desvio lateral de ombros ( $r=0,03 ; p=0,900)$, com o desvio de ombros projetados anteriormente $(r=-0,20$; $p=0,448)$, com o aumento da curvatura lombar ( $r=-0,25$; $p=0,341)$, com joelhos hiperestendidos $(r=0,03$; $p=0,900)$. Assim como também o tempo como surfista profissional não apresentou correlação significativa com esses desvios $(r=0,07$ e $p=0,779 ; r=-0,41$ e $p=0,105 ; r=0,05$ e $p=0,835 ; r=0,07$ e $p=0,779)$, respectivamente.

Não foi encontrada correlação significativa entre o ICP e o tempo de prática do surfe $(r=-0,29 ; p=0,262)$ e o tempo como surfista profissional $(r=-0,33 ; p=0,190)$.

\section{DISCUSSÃo}

De acordo com a classificação do método PSU, todos os surfistas profissionais apresentaram ICP maior que 65\% e, dessa forma, uma boa postura corporal. Resultados parecidos foram encontrados no estudo de Peirão et al. ${ }^{10}$ com 7 surfistas amadores (ICP=83,6 $\pm 4,8 \%)$.

Com o objetivo de analisar as relações entre desvios posturais, tempo de prática, número de lesões e magnitudes de impacto no joelho e no tornozelo na fase de aterrissagem do arremesso em suspensão no handebol em 9 atletas de uma equipe universitária, Santos et al. ${ }^{17}$ constataram que os atletas caracterizavam-se por joelhos semi-flexionados e pés abduzidos, e que as magnitudes de impacto podem ser consideradas como um mecanismo de lesão, bem como um fator interveniente nas alterações posturais dos atletas de handebol. Prati \& Prati ${ }^{\mathbf{1 8}}$ analisaram os níveis de aptidão física e tendências posturais de 11 bailarinas clássicas com mais de sete anos de prática, através do método PSU. Constataram um ICP médio de $86,9 \%$ e tendência a desvios na RCP
(13\% cifose), RCDL (8\% hiperlordose), RAQ (13\% abdômen proeminente e desnível de quadril) e RMI (18\% pés planos). Em outro estudo que utilizou o mesmo método de avaliação postural (PSU), objetivando investigar as possíveis alterações posturais em atletas das seleções brasileiras masculina $(n=15)$ e feminina $(n=16)$ de hóquei sobre a grama, foi constatado que grande parte dos atletas não apresentava desvios posturais. No entanto, dentre os que apresentaram, a maior prevalência foi na região de abdômen e quadril para os homens e na região de cabeça e pescoço para as mulheres ${ }^{\mathbf{1 2}}$. Os autores dos estudos acima sugerem que as tendências posturais detectadas em praticantes das respectivas atividades podem ser decorrentes da repetição de movimentos técnicos e da carga de treinamento, característicos de cada esporte, ao longo de anos de prática.

Os resultados percentuais das regiões $R C P, R C D L, R A Q$ e RMI de todos os surfistas profissionais apresentaram-se acima dos valores recomendados pelo método PSU. As regiões da cabeça e pescoço $(R C P)$ e dos membros inferiores (RMI) apresentaram as menores médias percentuais entre os atletas. No estudo de Peirão et al. ${ }^{10}$ com surfistas amadores, a RCP também apresentou o menor percentual $(76,0 \pm 9,6 \%)$ e o mesmo aconteceu no estudo de Quites et al. ${ }^{19}$ com trinta surfistas recreacionais $(63,3 \%)$, indicando uma maior incidência de desvios posturais para esta região quando comparadas com as demais, em surfistas recreacionais, profissionais e amadores. Entre os desvios mais presentes nesta região (RCP) estão o desvio anterior e o lateral de ombros, os quais foram observados nos resultados do presente estudo e no estudo de Peirão et al. ${ }^{\mathbf{1 0}}$, onde sete dos oito surfistas amadores apresentaram ambos os desvios. No estudo de Luz $^{20}$ com 16 bodyboardings, sete apresentaram ombros ligeiramente desviados para frente e 15 apresentaram um dos ombros um pouco mais elevado que o outro. Agra ${ }^{21}$ encontrou desvio lateral de ombros em cinco dos oito surfistas avaliados.

Entre os desvios da RMI apresentados pelo método PSU, encontra-se a hiperextensão dos joelhos. De acordo com os resultados do presente estudo, este desvio foi encontrado em 76,5\% dos surfistas e, no estudo de Peirão et al. ${ }^{10}$, quatro dos sete surfistas amadores apresentaram este desvio, assim como na pesquisa de Quites et al. ${ }^{19}$, em 28 dos 30 surfistas avaliados.

A terceira região corporal dos surfistas mais acometida neste estudo foi a abdominal e quadris (RAQ), sendo que $70,6 \%$ dos surfistas apresentaram aumento da curvatura lombar. No estudo de Agra $^{\mathbf{2 1}}$ com oito surfistas recreacionais, quatro apresentaram hiperlordose cervical e dois hiperlordose lombar. Peirão et al. ${ }^{10}$ constataram aumento da lordose lombar em seis dos sete surfistas. Luz ${ }^{\mathbf{2 0}}$ observou este desvio em 15 dos 16 bodyboardings avaliados.

No estudo de Souza et al. ${ }^{\mathbf{2 2}}$, com o objetivo de identificar a prevalência de lombalgia entre surfistas amadores, no 
município do Rio de Janeiro, por meio de um questionário epidemiológico (Quebec Back Pain Disability Scale) aplicado em 88 surfistas (grupo experimental) e 88 indivíduos não-surfistas (grupo controle), constatou-se uma maior prevalência de lombalgia entre os praticantes de surfe amadores quando comparados ao grupo controle $(p<0,05)$. 0 mesmo resultado foi encontrado por Trindade ${ }^{\mathbf{2 3}} \mathrm{em} 10$ dos 14 surfistas avaliados da cidade de Florianópolis.

O surfe possui três fases cíclicas: a remada, o período de espera e o surfar de pé sobre a prancha. De acordo com o estudo de Brasil et al. ${ }^{1}$ com surfistas recreacionais, a remada ocupou $54,4 \%$ do tempo total, o período de espera (parados) 27,8\%, surfando de pé sobre a prancha 3,7\% do tempo total, e outros movimentos 5,1\%. Resultados parecidos foram encontrados por Mendez-Villanueva et al. ${ }^{24}$ durante baterias de competições profissionais (World Qualifying Series - WQS), onde 51\% do tempo total foram dedicados à remada, $42 \%$ ao período de espera, $4 \%$ ao surfar de pé sobre a prancha e $2 \%$ a outros movimentos. De acordo com estes dados e com os resultados encontrados neste estudo e na literatura pesquisada, o desvio anterior de ombros em surfistas pode ser decorrente dos constantes movimentos de flexão dos braços durante a remada, realizados pelos músculos peitoral maior, redondo maior, grande dorsal e deltóide, que provocaria um encurtamento da musculatura envolvida. Possivelmente as irregularidades no alinhamento corporal, como a hiperextensão dos joelhos, também podem ocorrer devido à execução da remada, pois durante este movimento é realizada a hiperextensão dos joelhos com o objetivo de facilitar o equilíbrio do surfista sobre a prancha. Já o aumento da curvatura lombar pode ser decorrente da posição de hiperextensão da coluna cervical e lombar durante a remada, adotada para facilitar a visão, iá que este movimento ocupa $54,4 \%$ do tempo total de uma sessão de surfe recreacional ${ }^{1}$ e $51 \%$ do tempo total de baterias de competições profissionais ${ }^{24}$.

De acordo com resultados desta pesquisa, pode-se constatar que a incidência de desvios posturais em surfistas profissionais é encontrada freqüentemente nas regiões da cabeça e pescoço (RCDL), membros inferiores (RMI) e abdômen e quadril (RAQ), como desvio anterior e lateral de ombros, aumento da curvatura lombar e hiperextensão dos joelhos. Embora a análise postural utilizando o método PSU não tenha identificado desvios posturais acentuados, observou-se que essas assimetrias provavelmente podem ser decorrentes das características da própria modalidade.

\section{REFERÊNCIAS}

1. Brasil FK, Andrade DR, Oliveira LC, Ribeiro MA, Matsudo VKR. Freqüência cardíaca e tempo de movimento durante o surfe recreacional - estudo piloto. Rev Bras Ciên Mov. 2001 ; 9(4):65-75.

2. Steinman J. Surf \& Saúde. Florianópolis: TAO - Instituto de Medicina do Esporte, Acupuntura e Meditação; 2003.
3. Mendez-Villanueva A, Bishop D. Physiological aspects of surfboard riding performance. Sports Med. 2005;35(1):55-70.

4. Stein MC. Análise cinética da técnica da remada em praticante de surf [monografia]. Porto Alegre: Pontifícia Universidade Católica do Rio Grande do Sul; 2005.

5. Osmond G, Phillips MG, O'Neill M. 'Putting up your dukes': statues social memory and duke paoa kahanamoku. Int J Hist Sport. 2006;23(1):82-103.

6. Zoltan TB, Taylor KS, Achar SA. Health issues for surfers. Am Fam Physician. 2005;71(12):2313-7.

7. Nathanson A, Bird S, Dao L, Tam-Sing K. Competitive surfing injuries: a prospective study of surfing-related injuries among contest surfers. Am J Sports Med. 2007;35(1):113-7.

8. Taylor DMCD, Bennett D, Carter M, Garewal D, Finch CF. Acute injury and chronic disability resulting from surfboard riding. J Sci Med Sport. $2004 ; 7(4): 429-37$

9. Nathanson A, Haynes P, Galanis D. Surfing injuries. Am J Emerg Med. $2002 ; 20(3): 155-60$

10. Peirão R, Tirloni AS, Reis DC, Coelho PB, Guglielmo LGA. Incidência de desvios posturais em surfistas amadores. Em: Congresso de ciências do desporto e educação física dos países de língua portuguesa, XII, 2008, Porto Alegre. Anais do XII Congresso de ciências do desporto e educação física dos países de língua portuguesa. Porto Alegre: UFRGS; 2008.

11. Santos JB, Moro ARP, Cezar MR, Reis PF, Luz JD, Reis DC. Descrição do método de avaliação postural de Portland State University. Rev Fisioter Bras. 2005;6(5)atualizações.

12. Detanico D, Reis DC. Características da postura estática em atletas das seleções brasileiras de hóquei sobre a grama. Em: $6^{\circ}$ Fórum Internacional de Esportes, 2007, Florianópolis. Anais do $6^{\circ}$ Fórum Internacional de Esportes, Florianópolis: UNESPORTE; 2007.

13. Nahas MV. Atividade física, saúde e qualidade de vida: conceitos e sugestões para um estilo de vida ativo. $2^{a}$ ed. Londrina: Midiograf; 2001.

14. Ribeiro CZP, Akashi PMH, Sacco ICN, Pedrienelli A. Relações entre alterações posturais e lesões do aparelho locomotor em atletas de futebol de salão. Rev Bras Med Esporte. 2003;9(2):91-7.

15. Neto Júnior J, Pastre CM, Monteiro HL. Postural alterations in male brazilian athletes who have participated in international muscular power competitions. Rev Bras Med Esporte. 2004;10(3):195-8.

16. Zito M. The adolescent athlete: a musculoskeletal update. J Orthop Sports Phys Ther. 1983;5:20-5.

17. Santos SG, Detanico D, Graup S, Reis DC. Relação entre alterações posturais, prevalência de lesões e magnitudes de impacto nos membros inferiores em atletas de handebol. Fit Perf J. 2007;6(6):388-93.

18. Prati SRA, Prati ARC. Níveis de aptidão física e análise de tendências posturais em bailarinas clássicas. Rev Bras Cineantropom Desempenho Hum. 2006;8(1):80-7.

19. Quites MP, Duarte MF, Quites AM. Problemas posturais em surfistas de Florianópolis [monografia]. Florianópolis: Universidade Federal de Santa Catarina; 1995

20. Luz JD. Avaliação postural em atletas de bodyboarding [monografia]. Florianópolis: Universidade Federal de Santa Catarina; 2004.

21. Agra TD. Desvios posturais x surfe: presença de desvios posturais em praticantes de surfe em Tramandaí. [atualizado em 2007 dez 14; acesso em 2008 fev 8] Disponível em: http://www.congressurf.com.br/site/baixar. php? cliente $=1$ \&arquivo $=38$.

22. Souza MCMG, Fonseca VS, Sá VWB. Prevalência de lombalgia em surfistas amadores. Rev Bras Fisioter. 2006;10(sup).

23. Trindade JN. Incidência de dores lombares em surfistas amadores da grande Florianópolis [monografia]. Florianópolis: Universidade do Estado de Santa Catarina; 2006.

24. Mendez-Villanueva A, Bishop D, Hamer P. Activity profile of world-class professional surfers during competition: a case study. J Strength Cond Res. $2006 ; 20(3): 477-82$

Recebido: 02/07/2008 - Aceito: 21/09/2008 